\title{
Groundstroke Tennis Test
}

\author{
David Siahaan ${ }^{1 *}$, Nurkadri ${ }^{1}$, Pangondian Hotliber Purba ${ }^{1}$ \\ ${ }^{1}$ Pendidikan Kepelatihan Olahraga, Universitas Negeri Medan, Indonesia \\ *Corresponding author.Email: davidsiahaan@unimed.ac.id, nurkadri171214@gmail.com, pangondianpurba@yahoo.co.id
}

\begin{abstract}
The study aims to examine groundstroke tennis test and evaluates groundstroke performance. Groundstroke is a forehand or backhand shot that is executed after the ball bounces once on the court, from the back of the tennis court to around the baseline. This study uses test-retest methods for relibilities and t-test differentiation analisist for validity. The population is 81 beginer tennis players in university level (18 \pm 2 years) participated in this study. A sample of 67 people were taken randomly from the population using Slovin technique. Data from measurements that were done twice were tested for normality, homogenity and linearity as a condition for testing validity and reliability. Data from measurements I and II are normally distributed, homogeneous and linear. Groundstroke tennis test is valid with $t_{\text {score }}>t_{\text {standar }}(187>1,67)$ and coefficient reliability is .821 . Groundstroke tennis test is valid and reliable advice to measure groundstroke tennis basic skill for beginer tennis player at university level. The recommendation is the groundstroke tennis test be used to evaluate beginner level tennis players.
\end{abstract}

\section{Keywords: Tennis, Groundstroke, Tennis Test}

\section{INTRODUCTION}

Tennis is one of the sports that is played by hitting the ball using a racket on a long field 23,77 meter, where the ball can be hit after bouncing or volley. Tennis sports have developed and many people play this sport both as a means to improve fitness, achieve higher performance or recreation. Tennis tournaments have been competed for both professionals and amateurs. Some tennis tournaments at senior professional level are Olympic, ITF National Circuit, ATP Challenger Tour, ATP World Tour 250 series, ATP World Tour 500 series, ATP World Tour Master 1000, ATP Final, Grand Slam. While tennis tournaments that are competed for juniors are grades 1-5, Grades C (International Team Competitions), Grades B (Regional Championships), Grades A (Including 4 Grand Slams). All types of tournaments are a medium of competing against athletes so that they can improve and maintain achievements to the highest level. "Tennis can be a sport of a lifetime for almost everyone can play at any age, do not need many people to play a game, do not need much money to play tennis, brings people together and great way to keep fit" [1]. In Indonesia tennis has become one of the sports that has developed which in almost every province there has been guidance for junior or senior tennis players in the form of Student Training Centers and coaching under the guidance of PELTI and tennis matches.

There are basic techniques or basic strokes to play tennis. They are groundstroke, lob, serve, overhead and volley. Groundstroke is the stroke when you hit a ball that has bounced off the ground. Groundstroke are the basic shots you have made once the point has begun. "Although these shots can be played from anywhere in the court, they are usually made from the baseline and after the ball has bounced" [2].

Groundstroke is one of the techniques most often done in tennis games especially in single games. Return service is one of the ground stroke techniques that must be mastered by players whose ball can be hit by the service recipient only after the ball bounces. In a single $80 \%$ match the technique used in the game is groundstroke. In addition to maintaining a defensive position and for attacking, tennis players can stroke from behind the back line to create opportunities to attack the opponent's defense by hitting the ball at a point that is difficult for the opponent to reach.

In training coaching and also tennis learning, accurate measurement of the ability to play tennis is needed, whether it be groundstroke, service, smash, volleyball and other techniques. Every good player for beginner, advanced and professional level needs to develop a standard measuring instrument to test the ability of the technique. The development of this tennis groundstroke test needs to be done to test the results of learning and training from beginner tennis players so that it can be identified as an increase in their abilities and the results can be an evaluation material both to the trainer and other parties in need.

Several measurement tests that previously existed have Hewitt's tennis achievement test. This test evaluates three basic skill tests in tennis at the university level - service, forehand and backhand drives with reliability levels between .75-94. "This test can be given to conduct evaluations in playing tennis to beginners, intermediate and varsity groups" [3]. The weakness of this test and 
measurement is that it does not measure the ability to play tennis dynamically, which is only to hit the ability of one of the playing techniques, forehand or backhand. Basically playing tennis is moving closer to the hitting ball on the opponent's field and again preparing to hit the ball again whether it's the ball in the backhand direction or in the forehand direction.

This test was developed to measure the ability of tennis players to hit with groundstroke or volleyball. Within 30 seconds the ability is measured by how much the trainer can hit the ball correctly on the wall. The validity of this test is .85-.92. While the reliability is .86 to .90 . The disadvantage of this test is that the ability is measured only by reflecting the ball onto the wall where the ball hit to the reflection wall has different characteristics from the ball being hit on the actual tennis court. The rotation and height of the ball certainly greatly affects the arrangement of the racquet's surface when hitting the ball so that the ball can still be hit on the playing field.

Tennis is carried out directly in the tennis court by using a throwing machine as a feeder. This test has 61 validity for beginners and .85 for intermediate players. Whereas test reliability has .80 for beginners and also intermediate classes. The deficiency of this test is also to measure the ability of forehand or backhand where the ability is measured not simultaneously. It is necessary to develop a test that directly measures the ability of groundstroke to measure forehand and backhand abilities simultaneously.

\section{METHOD}

"The method of this research is a development method, which refers to Sugiono" [4]. The groundstrokes tennis test were developed with reference to the need to measure the technical capability groundstrokes in the game of tennis. The research is held on the outdoor tennis court State University of Medan. The surface of the field that used for groundstroke tennis test is a type of hard court (granite). The population of this research is 87 beginner tennis players. "A sample of 67 people was obtained by the Slovin formula" [5]. The research subjects are beginner tennis players who have grown up where their age is 17-19 years. The population daily activities of the research subject are studying at the sports science faculty where they carry out physical activities every day and have good psychomotor abilities.

Testing the validity and reliability of the measuring instrument on the groundstroke was carried out twice where I and II or test and retest were performed. After the measurement is done, the analysis of the requirements analysis is carried out so that it meets the parametric testing. The conditions that must be met are samples taken with a random technique, the data to be tested must be homogeneous, normal distribution and linear. Furthermore, after these conditions are met, the results of each measurement are tested by product moment correlation test to determine the validity of the test tool, namely knowing the strength of the relationship between each measurement I and II. The purpose of this test is to measure whether this tools is appropriate for measuring abilities groundstroke tennis. Then test reliability to find out whether as says are groundstroke tennis consistent in measuring the technique. After the analysis requirements test is fulfilled, the validity and reliability tests are carried out, then proceed with making the norm reference on the test tools groundstroke tennis.

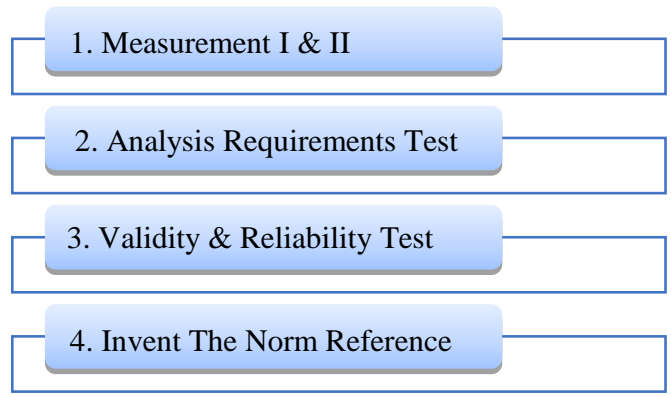

Figure 1: Steps in Examine The Test Tools Groundstroke Tennis.

\section{RESULTS AND DISCUSSION}

\subsection{Description of Measurement Results}

In the first measurement of 81 students who performed tennis groundstroke test as many as 15 people obtained 4-7 groundstroke abilities, 12 people obtained a figure of 8-11, 13 people obtained 12-15 abilities, 12 people obtained groundstroke abilities from $16-19,15$ people obtained groundstroke abilities from 20-23, 11 people obtained groundstroke abilities from 24 to 27,11 people obtained groundstroke abilities from 28 to 31 .

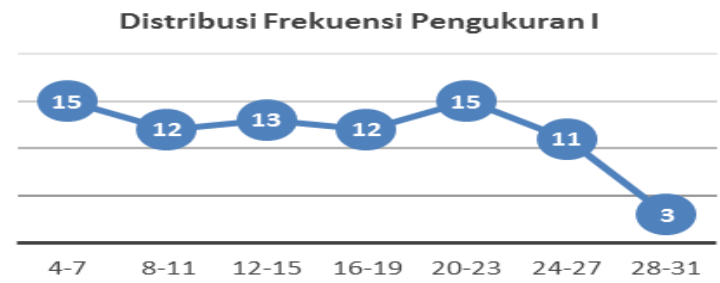

Figure 2 : Chart of Data From Seccond Measurement.

In the measurement of the seccond tennis groundstroke tests for 81 students there were 7 people obtained 2-7 groundstroke skills, 16 people gained $8-13,22$ people gained abilities $14-19,16$ people gained $20-25,11$ people gain groundstroke abilities from 26 to 31,6 people gain 32-37 groundstroke abilities, 3 people gain 38-43 groundstroke abilities.

Distribusi Frekuensi Pengukuran II

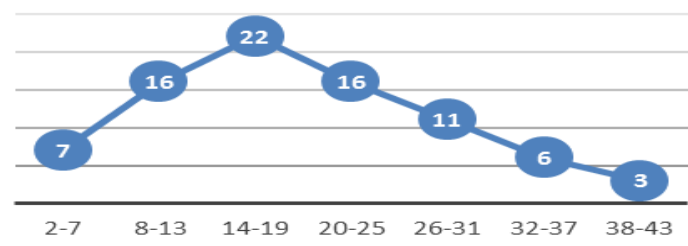

Figure 3 : Chart of Data From Second Measurement. 


\subsection{Analysis Requirements Test}

In using the parametric statistical test, it is necessary to carry out analysis requirements test or classic violation test. This test requires several things, namely the normality test, homogeneity test and linearity test, besides that the data analyzed in the parametric statistical test must be interval and ratio scale and the sampling is done randomly (Supriadi. U. S 2013)

Tabel 1. The Result of Analysis Requirements Test.

\begin{tabular}{|l|l|l|c|}
\hline Data & Homogenity & Normality & $\begin{array}{l}\text { Linearit } \\
\mathrm{y}\end{array}$ \\
\hline Test I & \multirow{2}{*}{$.076>.05}$. & $180>.05$ & \multirow{2}{*}{.283} \\
\cline { 1 - 1 } \cline { 3 - 3 } Test II & & $062>.05$ & \\
\hline
\end{tabular}

The results of the homogeneity test with the test result which if the probability value (sig)> .05 then the data of the two groups is homogeneous (Kadir: 2015) The value of sig .076 is obtained where compared with the value of .05 then $.076>.05$ so that it can be concluded that the data comes from the same or homogeneous variant.

The results of the normality test with kolmogorov smirnov which if the probability value (sig,)> .05 then the data is normally distributed (Kadir: 2015). Obtained sig value. In measurement I. 180 and in measurement II .062. when compared to .05 , the data in measurement $\mathrm{I}$ are normally distributed $(.180>.05)$. whereas in measurement II the data is also normally distributed because of .062> .05 .

Linearity tests are conducted to determine whether two variables have a significant relationship or not. The results of the linearity test on measurement I and measurement II obtained the sig value. 283 . Then it can be concluded that the two data groups have a significant linear relationship because of .283> .05.

After the requirements for the parametric test are fulfilled, the test can be continued with validity and reliability test. Validity test results with distinguishing analysis. The steps to carrying out this test are to distinguish the highest $27 \%$ score and the lowest $27 \%$ score after all the data is sorted. After the two data are obtained, then proceed with the unpaired t test. The results of the analysis show that $t_{\text {score }}=187$ while $t_{\text {standard }}=1.67$. It can be concluded that the groundstroke tennis test is valid and feasible to use to measure the groundstroke ability of novice tennis players at the student level.

Reliability test results with test and retest methods. After the data is obtained, it is tested using product moment correlation to test the relationship between two variables. The results of the analysis obtained the value $r$. 821. This value is included in the category of very strong relationships. It can be concluded that the developed tennis groundstroke test is reliable, namely that measurements made on the beginner's groundstroke ability are consistent or can be repeated. After the tennis groundstroke test is declared valid and reliable then proceed with making a norm reference to make an evaluation of the results of tennis groundstroke.

\subsection{The Groundstroke Tennis Test}

Description: groundstroke test aims to measure the ability of the groundstroke to play tennis both forehand and backhand simultaneously. This test is precise and consistent in measuring the technique. This test is intended for novice tennis players both men and women at the university level but can also be done for junior beginer players at other ages. The target consists of 1-5 points where the closer the baseline the number is higher. The distance between points $2-5$ is 1,345 meters or 4 feet 6 inches.

Validity authenticity: As for the results of the validity test, it is known that the tennis groundstroke test developed is valid with a value of $t_{\text {score }}=187$ while thet $_{\text {standard }}=1.67$. while the reliability test performed obtained by the reliability coefficient is .821 shows a very strong relationship between the test and retest done.

Administrative Feasibility: it takes 20 to 30 minutes to measure 30-40 students using one or two test fields. Personel needed at the time of the test are one person ball feeders at each test field, score keeper and some people collect balls. It is given the opportunity for each tennis player to warm up to do a 10-15 minute groundstroke with his friend. The tools needed are the boundary line markers of the target between the left and right lines. The marker must be able to clearly limit the target at each point to the target when viewed from the baseline where the tertiary stand. Basket tennis ball, tennis rackets with balls are available as many as needed. To see the point more clearly a score board can be made on the value of each target starting from the values of 1 to 5 , so that the athlete knows how much he gets on each hit made.

Direction: a feeder stands in the middle of the net front of the target area. His job is to feed the ball correctly against tests. The ball is alternately hit in the direction of the forehand and backhand, where it is right at the marked feeding target. The height of the reflection between the waist and shoulder tests. Tennis players hit the ball starting from the forehand direction towards the set target. Furthermore, moving to hit the ball in the backhand direction is done repeatedly for up to 10 punches. Warming can be done before the player performs a test which is hitting the ball repeatedly 4 times which is in the direction of the forehand and two in the direction of the backhand.

Scoring Authenticity: Each hit ball is given a value where the ball bounces. The ball that is hit out or is connected on the net does not get a value. Of the 10 strokes, the total value obtained by each player is added. Then to evaluate the results can be converted to the norm test for tennis tennis courts. 
Table: Reference Norms in Assessment Standards.

\begin{tabular}{cccc}
\hline Scale & Score Limit & $\begin{array}{c}\text { Score } \\
\text { Range }\end{array}$ & Value \\
\hline$\dot{\mathrm{X}}+2.4 * \mathrm{~S}$ & $38>$ & 38 & 10 \\
$\dot{\mathrm{X}}+1.8 * \mathrm{~S}$ & 32 & $32-37$ & 9 \\
$\dot{\mathrm{X}}+1.2 * \mathrm{~S}$ & 27 & $27-31$ & 8 \\
$\dot{\mathrm{X}}+0.6 * \mathrm{~S}$ & 24 & $24-26$ & 7 \\
$\dot{\mathrm{X}}+0.0 * \mathrm{~S}$ & 17 & $17-23$ & 6 \\
$\dot{\mathrm{X}}-0.6 * \mathrm{~S}$ & 11 & $11-16$ & 5 \\
$\dot{\mathrm{X}}-1.2 * \mathrm{~S}$ & 7 & $7-10$ & 4 \\
$\dot{\mathrm{X}}-1,8 * \mathrm{~S}$ & 2 & $2-6$ & 3 \\
$\dot{\mathrm{X}}-2,4 * \mathrm{~S}$ & 0 & $<2$ & 2 \\
\hline
\end{tabular}

Table: Norms of Tests Groundstroke Tennis

\begin{tabular}{cc}
\hline Norma & Rentang \\
\hline Very Good & $>32$ \\
Good & $24-31$ \\
Fair & $11-23$ \\
Poor & $7-16$ \\
Very Good & $<7$ \\
\hline
\end{tabular}

Additional Comment: Assessment of results for forehand groundstrokes and backhand drives is carried out by performance where the ball is fed across the net by the feeder or feeder to a predetermined point. Students move to hit the ball starting from forehand, backhand, alternately up to 10 repetitions ( 5 forehands and 5 backhand). The number of values obtained in 10 times hitting the ball is summed and the value becomes the value of the ability of students to do a groundstroke. A ball that is struck out of the field and involves on the net not entering the playing field does not get a score.

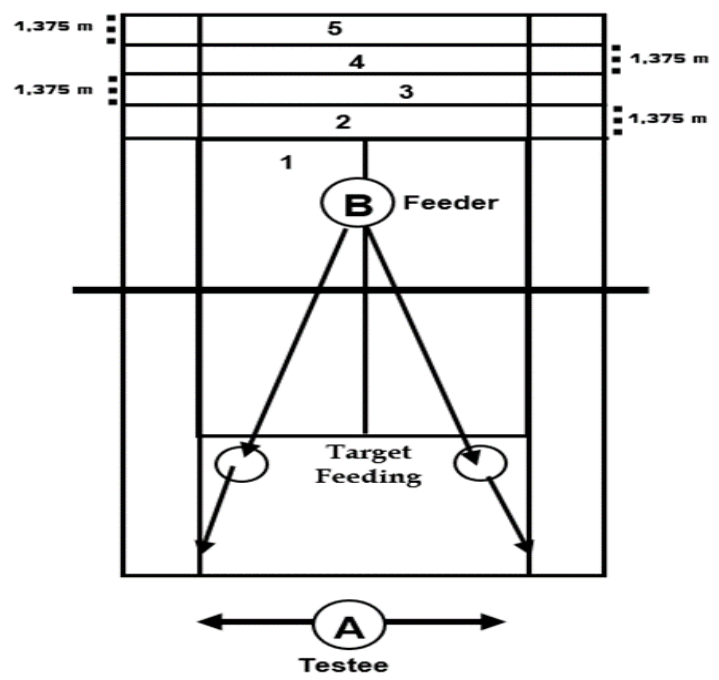

Figure 4: Grounstroke Tennis Test

\section{CONCLUSION}

The results of the validity and reliability analysis show that tennis groundstroke tests are feasible to be used as a measuring instrument or instrument to measure groundstroke tennis's ability in university-level beginner players. So to evaluate the ability of groundstroke tennis can be done with this test.

\section{REFERENCES}

[1] Patric and P. Bodo. "Tennis For Dummies". Indianapolis: Wiley Publishing, Inc.1998. pp. 78.

[2] D. Ray and P. Hodges. "A Comprehensive Guide to Sport Skills Tests and Measurement". Illionist: Charles C Thomas Publisher.1978. pp. 25.

[3] J. Hewitt. "Wuarterly review. American Associant for Health, Phusical Education and Recreation" Vol. 37. No. 2. 1966. pp. 231-240.

[4] Juliansyah. "Research methodology". Jakarta: Prenadamedia Group. 2015. Pp. 47.

[5] F. Rolf. "School success in the Go Tennis test". Great Britain: Dorling Kindersley. 2006. Pp. 87.

[6] Sugiyono. "Educational Research Methods Quantitative, Qualitative and R \& D Approaches". Bandung: Alfabeta. 2013. pp. 28. 